\title{
Local Smooth Solution and Non-Relativistic Limit of Radiation Hydrodynamics Equations
}

\author{
Jianwei Yang, ${ }^{1}$ Shu Wang, ${ }^{2}$ and Yong $\mathrm{Li}^{2}$ \\ ${ }^{1}$ College of Mathematics and Information Science, North China University of Water Resources and \\ Electric Power, Zhengzhou 450011, China \\ ${ }^{2}$ College of Applied Sciences, Beijing University of Technology, PingLeYuan100, Chaoyang District, \\ Beijing 100022, China
}

Correspondence should be addressed to Jianwei Yang, yjw@emails.bjut.edu.cn

Received 5 May 2010; Accepted 16 July 2010

Academic Editor: Donal O’Regan

Copyright (C) 2010 Jianwei Yang et al. This is an open access article distributed under the Creative Commons Attribution License, which permits unrestricted use, distribution, and reproduction in any medium, provided the original work is properly cited.

\begin{abstract}
We investigate a multidimensional nonisentropic radiation hydrodynamics model. We study the local existence and the convergence of the nonisentropic radiation hydrodynamics equations via the non-relativistic limit. The local existence of smooth solutions to both systems is obtained. For well-prepared initial data, the convergence of the limit is rigorously justified by an analysis of asymptotic expansion, an energy method, and an iterative scheme. We also establish uniform a priori estimates with respect to $\epsilon$.
\end{abstract}

\section{Introduction}

In this paper, we study a system of PDEs describing radiation-driven perfect compressible flows, in particular in astrophysics (cf. [1-4]). Assuming that the radiative temperature and the fluid temperature are equal, and that the gas is radiatively opaque so that the equilibrium diffusion will be dealt with, and the mean free path of photons is much smaller than the typical length of the flow, then, we can write the equations of radiation hydrodynamics without radiative heat diffusivity in $\mathbb{R}^{\mathrm{d}}$, describing the conservation of mass, momentum and energy, as (see $[2,3,5])$

$$
\begin{gathered}
\partial_{t} \rho+\operatorname{div}(\rho \mathbf{u})=0 \\
\partial_{t}(\rho \mathbf{u})+\operatorname{div}(\rho \mathbf{u} \otimes \mathbf{u})+\nabla\left(p+\frac{1}{3} \epsilon \theta^{4}\right)=0 \\
\partial_{t} E+\operatorname{div}\left[\left(E+p+\frac{1}{3} \epsilon \theta^{4}\right) \mathbf{u}\right]=0
\end{gathered}
$$


for $(x, t) \in \mathbb{R}^{3} \times[0, T), T>0$, where $\rho, \mathbf{u}=\left(u_{1}, \ldots, u_{d}\right)^{T}, p$, and $\theta$ denote the density, velocity, thermal pressure, and absolute temperature, respectively, $\epsilon=8 \pi^{5} k^{4} / 15 h^{3} c^{3}>0$ is a radiation constant, and $c$ is the light speed, and

$$
E=\frac{1}{2} \rho \mathbf{u}^{2}+\rho e+\epsilon \theta^{4}
$$

is the total energy, $e=e(\rho, \theta)$ is the internal energy, and $\mathbf{u}^{2}=\sum_{i=1}^{d} u_{i}^{2}$ is the square of the macroscopic velocity.

From (1.1) and (1.2), we see that the system includes both gas and radiative contributions to flow dynamics. The quantities $(1 / 3) \epsilon \theta^{4}$ and $\epsilon \theta^{4}$ represent the radiative pressure and radiative energy density, respectively. To complete system (1.1), one needs the equation of state for the pressure $p=p(\rho, \theta)$. In this paper, for the purpose of our test problems, we will limit our study to the polytropic ideal gases, namely: $p=R \rho e=(\gamma-1) \rho e$ with $\gamma>1$ being the specific heat ratio and $e=c_{V} \theta$ with $c_{V}$ being the specific heat; we assume $c_{V}=1$ without loss of generality.

We point out that if one assumes $\epsilon \rightarrow 0$ in (1.1), then system (1.1) reduces to the usual inviscid Euler equations:

$$
\begin{gathered}
\partial_{t} \rho^{0}+\operatorname{div}\left(\rho^{0} \mathbf{u}^{0}\right)=0, \\
\partial_{t}\left(\rho^{0} \mathbf{u}^{0}\right)+\operatorname{div}\left(\rho^{0} \mathbf{u}^{0} \otimes \mathbf{u}^{0}\right)+\nabla p^{0}=0, \\
\partial_{t} E^{0}+\operatorname{div}\left[\left(E^{0}+p^{0}\right) \mathbf{u}^{0}\right]=0,
\end{gathered}
$$

which are nonisentropic and compressible Euler equations.

The aim of this paper is to justify rigorously the local existence of smooth solutions of system (1.1) and the convergence of system (1.1) to this formal limit equations (1.3).

Concerning the non-relativistic limit $c \rightarrow \infty$, that is, $\epsilon \rightarrow 0$, there are only partial results. Indeed, we know that the phenomenon of non-relativistic is important in many physical situations involving various nonequilibrium processes. For example, important examples occur in inviscid radiation hydrodynamics [6], in quantum mechanics [7], in KleinGordon-Maxwell system [8], in Vlasov-Poisson system [9], in Euler equations [10], in EulerMaxwell equations [11, 12], and so on.

In this paper, we are interested in the nonrelativistic limit $\epsilon \rightarrow 0$ in the problem (1.1) for the radiation hydrodynamics equations. We prove the existence of smooth solutions to the problem (1.1) and their convergence to the solutions of the compressible and nonisentropic Euler equations in a time interval independent of $\epsilon$. For this propose, we use the method of iteration scheme and classical energy method. The convergence of the radiation hydrodynamics equations to the compressible and nonisentropic Euler equations is achieved through the energy estimates for error equations derived from (1.1) and it's formal limit equations (1.3).

The remainder of this paper is arranged as follows: In the next section, we give the local smooth solutions to both system (1.1) and (1.3). Section 3 is devoted to justify the convergence of (1.1) to (1.3). By formal analysis, we show that the leading profiles of the density, velocity, and temperature with respect to $\epsilon$ satisfy a compressible nonisentropic Euler equations, and their next order profiles satisfy the corresponding linearized equations. 
The Cauchy problem for this nonisentropic Euler equations is solved in this section. The final part is devoted to rigorously justifying the asymptotic expansion developed in Section 3 and obtaining the convergence of solutions to the multidimensional compressible nonisentropic Euler system in a time interval independent of $\epsilon$.

\section{Notations and Preliminary Results}

(1) Throughout this paper, $\nabla=\nabla_{x}$ is the gradient, $\alpha=\left(\alpha_{1}, \ldots, \alpha_{d}\right)$ and $\beta$ are multiindeices, and $H^{s}\left(\mathbb{R}^{d}\right)$ denotes the standard Sobolev's space in $\mathbb{R}^{d}$, which is defined by Fourier transform, namely, $f \in H^{s}\left(\mathbb{R}^{d}\right)$ if and only if

$$
\|f\|_{s}^{2}=(2 \pi)^{d} \sum_{k \in \mathfrak{Z}^{d}}\left(1+|k|^{2}\right)^{s}|(\mathcal{F} f)(k)|^{2}<+\infty
$$

where $(\mp f)(k)=\int_{\mathbb{R}^{d}} f(x) e^{-i k x} d x$ is the Fourier transform of $f \in H^{s}\left(\mathbb{R}^{d}\right)$.

(2) Also, we need the following basic Moser-type calculus inequalities (see, Klainerman and Majda $[13,14])$ : for $f, g, v \in H^{s}$ and any nonnegative multi-index $\alpha,|\alpha| \leq s$,

$$
\begin{gathered}
\left\|D_{x}^{\alpha}(f g)\right\|_{L^{2}} \leq C_{s}\left(\|f\|_{L^{\infty}}\left\|D_{x}^{s} g\right\|_{L^{2}}+\|g\|_{L^{\infty}}\left\|D_{x}^{s} f\right\|_{L^{2}}\right), \quad s \geq 0, \\
\left\|D_{x}^{\alpha}(f g)-f D_{x}^{\alpha} g\right\|_{L^{2}} \leq C_{s}\left(\left\|D_{x} f\right\|_{L^{\infty}}\left\|D_{x}^{s-1} g\right\|_{L^{2}}+\|g\|_{L^{\infty}}\left\|D_{x}^{s} f\right\|_{L^{2}}\right), \quad s \geq 1, \\
\left\|D_{x}^{s} A(v)\right\|_{L^{2}} \leq C_{s} \sum_{j=1}^{s}\left\|D_{x}^{s} A(v)\right\|_{L^{\infty}}\left(1+\|\nabla v\|_{L^{\infty}}\right)^{s-1}\left\|D_{x}^{s} v\right\|_{L^{2}}, \quad s \geq 1 .
\end{gathered}
$$

(3) (Sobolev's inequality). For $s>d / 2$,

$$
\|f\|_{L^{\infty}} \leq C_{s}\|f\|_{s}
$$

(4) If $s>d / 2$, then for $f, g \in H^{s}$ and $|\alpha| \leq s$,

$$
\left\|D_{x}^{\alpha}(f g)\right\|_{L^{2}} \leq C_{s}\|f\|_{s}\|g\|_{s}
$$

\section{The Local Existence}

In this section, we give our main result about local existence. For this purpose, we first rewrite the system (1.1) as a symmetric hyperbolic system of first order. Then, we prove the local existence and uniqueness of smooth solutions to the Cauchy problem for (1.1). 
For smooth solutions, the system (1.1) can be rewritten as follows:

$$
\begin{gathered}
\partial_{t} \rho+\operatorname{div}(\rho \mathbf{u})=0, \\
\partial_{t} \mathbf{u}+(\mathbf{u} \cdot \nabla) \mathbf{u}+\frac{R \theta}{\rho} \nabla \rho+\left(R+\frac{4 \epsilon \theta^{3}}{3 \rho}\right) \nabla \theta=0, \\
\partial_{t} \theta+(\mathbf{u} \cdot \nabla) \theta+\left(R \theta+\frac{(4 / 3-4 R) \epsilon \theta^{4}}{\rho+4 \epsilon \theta^{4}}\right) \operatorname{div} \mathbf{u}=0 .
\end{gathered}
$$

In fact, (2.1) is a non-relativistic, non-isotropic, and compressible Euler equations.

For convenience, we introduce the following two functions:

$$
\begin{aligned}
& f_{1}(\rho, \theta)=\frac{4 \theta^{3}}{3 \rho} \\
& f_{2}(\rho, \theta)=\frac{(4 / 3-4 R) \theta^{4}}{\rho+4 \epsilon \theta^{4}} .
\end{aligned}
$$

Then, (2.1) can be rewriten as follows:

$$
\begin{gathered}
\partial_{t} \rho+\operatorname{div}(\rho \mathbf{u})=0, \\
\partial_{t} \mathbf{u}+(\mathbf{u} \cdot \nabla) \mathbf{u}+\frac{R \theta}{\rho} \nabla \rho+\left(R+f_{1} \epsilon\right) \nabla \theta=0, \\
\partial_{t} \theta+(\mathbf{u} \cdot \nabla) \theta+\left(R \theta+f_{2} \epsilon\right) \operatorname{div} \mathbf{u}=0 .
\end{gathered}
$$

Denote the vector and matrix

$$
V=(\rho, \mathbf{u}, \theta)^{T}, \quad \tilde{A}_{j}(V)=u_{j} I_{(d+2) \times(d+2)}+\left(\begin{array}{ccc}
0 & \rho e_{j}^{T} & 0 \\
\frac{R \theta}{\rho} e_{j} & 0 & \left(R+f_{1} \epsilon\right) e_{j} \\
0 & \left(R \theta+f_{2} \epsilon\right) e_{j}^{T} & 0
\end{array}\right)
$$

where $\left(e_{1}, \ldots, e_{d}\right)$ is the canonical basis of $\mathbb{R}^{d}$ and $y_{i}$ denotes the $i$ th component of $y \in \mathbb{R}^{d}$. Thus, we can rewrite the system (2.3) as follows:

$$
\partial_{t} V+\sum_{j=1}^{d} \tilde{A}_{j}(V) \partial_{x_{j}} V=0
$$

We will study the Cauchy problem for (2.5) together with the initial data

$$
V(x, 0)=V_{0}(x), \quad x \in \mathbb{R}^{d} .
$$


It is not difficult to see that the equations of $V$ in (2.5) are symmetrizable and hyperbolic. If we introduce the $(d+2) \times(d+2)$ matrix

$$
A_{0}(V)=\left(\begin{array}{ccc}
\rho^{-1} & 0 & 0 \\
0 & \frac{\rho}{R \theta} & 0 \\
0 & 0 & \frac{\rho\left(R+f_{1} \epsilon\right)}{R \theta\left(R \theta+f_{2} \epsilon\right)}
\end{array}\right) \text {, }
$$

which is positive definite for $\epsilon \ll 1$, then $A_{j}(V)=A_{0}(V) \tilde{A}_{j}(V)$ are symmetric for all $1 \leq j \leq d$. Note that for smooth solutions, (2.3) is equivalent to that of (2.5).

Noticing the above facts and using the standard iteration techniques of local existence theory for symmetrizable hyperbolic system (see [15]), we have the following.

Theorem 2.1. Assume that $V_{0} \in H^{s}, s>d / 2+1, V_{0}(x) \in G_{1}, \overline{G_{1}} \subset \subset \in=\left\{V: \rho, \theta \geq C_{1}>0\right\}$, and $C_{1}$ is a positive constant. Then there exists a time interval $[0, T]$ with $T>0$, such that (2.5) and (2.6) have a unique solution $V(x, t) \in C^{1}\left(\mathbb{R}^{d} \times[0, T]\right)$, with $V(x, t) \in G_{2}, G_{2} \subset \subset G$ for $(x, t) \in \mathbb{R}^{d} \times[0, T]$. Furthermore, $V \in C\left([0, T], H^{s}\right) \cap C^{1}\left([0, T], H^{s-1}\right)$, and $T$ depends on $\epsilon,\left\|V_{0}\right\|_{s}$ and $G_{1}$.

\section{Asymptotic Analysis}

\subsection{Formal Asymptotic Expansions}

Let $\left(\rho^{\epsilon}, \mathbf{u}^{\epsilon}, \theta^{\epsilon}\right)$ be the smooth solution to the system (2.3). In this section, we are going to study the formal expansions of $\left(\rho^{\epsilon}, \mathbf{u}^{\epsilon}, \theta^{\epsilon}\right)$ as $\epsilon \rightarrow 0$. To this end, we assume that initial data $\left(\rho_{0}^{\epsilon}, \mathbf{u}_{0}^{\epsilon}, \theta_{0}^{\epsilon}\right)$ have the asymptotic expansion with respect to $\epsilon$ :

$$
\begin{aligned}
& \rho_{0}^{\epsilon}(x)=\sum_{j=0}^{m} \epsilon^{j} \rho_{j}(x)+\epsilon^{m+1} \rho_{m+1}^{\epsilon}(x), \\
& \mathbf{u}_{0}^{\epsilon}(x)=\sum_{j=0}^{m} \epsilon^{j} \mathbf{u}_{j}(x)+\epsilon^{m+1} \mathbf{u}_{m+1}^{\epsilon}(x), \\
& \theta_{0}^{\epsilon}(x)=\sum_{j=0}^{m} \epsilon^{j} \theta_{j}(x)+\epsilon^{m+1} \theta_{m+1}^{e}(x) .
\end{aligned}
$$

Then, we take the following ansatz:

$$
\begin{aligned}
& \rho^{\epsilon}(x, t)=\sum_{j \geq 0} \epsilon^{j} \rho^{j}(x, t), \\
& \mathbf{u}^{\epsilon}(x, t)=\sum_{j \geq 0} \epsilon^{j} \mathbf{u}^{j}(x, t), \\
& \theta^{\epsilon}(x, t)=\sum_{j \geq 0} \epsilon^{j} \theta^{j}(x, t),
\end{aligned}
$$


in terms of $\epsilon$ for the solutions to the system (2.3). Substituting the expansion (3.2) into the system (2.3), we have the following.

(1) The leading terms $\left(p^{0}, \mathbf{u}^{0}, \theta^{0}\right)$ satisfy the following problem:

$$
\begin{gathered}
\partial_{t} \rho^{0}+\operatorname{div}\left(\rho^{0} \mathbf{u}^{0}\right)=0, \\
\partial_{t} \mathbf{u}^{0}+\left(\mathbf{u}^{0} \cdot \nabla\right) \mathbf{u}^{0}+\frac{R \theta^{0}}{\rho^{0}} \nabla \rho^{0}+R \nabla \theta^{0}=0, \\
\partial_{t} \theta^{0}+R \theta^{0} \operatorname{div} \mathbf{u}^{0}+\left(\mathbf{u}^{0} \cdot \nabla\right) \theta^{0}=0, \\
\left(\rho^{0}, \mathbf{u}^{0}, \theta^{0}\right)(t=0)=\left(\rho_{0}, \mathbf{u}_{0}, \theta_{0}\right) .
\end{gathered}
$$

These are nonisentropic and compressible Euler equations of ideal fluids. In fact, (3.3) is equivalent to (1.3).

(2) For any $j \geq 1$, the profiles $\left(\rho^{j}, \mathbf{u}^{j}, \theta^{j}\right)$ satisfy the following problem for linearized equations:

$$
\begin{gathered}
\partial_{t} \rho^{j}+\sum_{k=0}^{j} \operatorname{div}\left(\rho^{k} \mathbf{u}^{j-k}\right)=0, \\
\partial_{t} \mathbf{u}^{j}+\sum_{k=0}^{j}\left(\mathbf{u}^{k} \cdot \nabla\right) \mathbf{u}^{j-k}+R\left(\theta^{j} \nabla \ln \rho^{0}+\theta^{0} \nabla\left(\ln ^{\prime} \rho^{0} \rho^{j}\right)+\nabla \theta^{j}\right)+g_{1}^{j-1}=0, \\
\partial_{t} \theta^{j}+\sum_{k=0}^{j}\left(\mathbf{u}^{k} \cdot \nabla\right) \theta^{j-k}+R \sum_{k=0}^{j} \theta^{k} \operatorname{div} u^{j-k}+g_{2}^{j-1}=0, \\
\left(\rho^{j}, \mathbf{u}^{j}, \theta^{j}\right)(t=0)=\left(\rho_{j}, \mathbf{u}_{j}, \theta_{j}\right),
\end{gathered}
$$

where $g_{i}^{0}=0(i=1,2)$ for $j \geq 1$. In fact, $g_{i}^{j-1}(i=1,2)$ depends only on $\left(\left\{\rho^{k}, u^{k}, \theta^{k}\right\}_{k \leq j-1}\right)$ and can be obtained from the following relation:

$$
\begin{aligned}
g_{1}^{j-1}= & \left.\frac{R}{j !} \frac{d^{j}}{d \epsilon^{j}}\left[\left(\left(\theta^{0}+\sum_{j \geq 1} \epsilon^{j} \theta^{j}\right) \nabla \ln \left(\rho^{0}+\sum_{j \geq 1} \epsilon^{j} \rho^{j}\right)\right)+f_{1}\left(\rho^{0}+\sum_{j \geq 1} \epsilon^{j} \rho^{j}, \theta^{0}+\sum_{j \geq 1} \epsilon^{j} \theta^{j}\right) \epsilon\right]\right|_{\epsilon=0} \\
& -R\left(\theta^{j} \nabla \ln \rho^{0}+\theta^{0} \nabla\left(\ln ^{\prime} \rho^{0} \rho^{j}\right)\right), \\
g_{2}^{j-1}= & \left.\frac{1}{j !} \frac{d^{j}}{d \epsilon^{j}}\left[f_{2}\left(\rho^{0}+\sum_{j \geq 1} \epsilon^{j} \rho^{j}, \theta^{0}+\sum_{j \geq 1} \epsilon^{j} \theta^{j}\right) \operatorname{div}\left(u^{0}+\sum_{j \geq 1} \epsilon^{j} u^{j}\right) \epsilon\right]\right|_{\epsilon=0} .
\end{aligned}
$$




\subsection{Determination of Formal Expansions}

\subsubsection{Preliminary}

From (3.4), we know that once $\left(\rho^{0}, \mathbf{u}^{0}, \theta^{0}\right)$ are solved from the problem (3.3), $\left(\rho^{1}, \mathbf{u}^{1}, \theta^{1}\right)$ are solutions to the following problem for a linearized equations:

$$
\begin{gathered}
\partial_{t} \rho^{1}+\operatorname{div}\left(\rho^{0} \mathbf{u}^{1}+\rho^{1} \mathbf{u}^{0}\right)=0, \\
\partial_{t} \mathbf{u}^{1}+\left(\mathbf{u}^{0} \cdot \nabla\right) \mathbf{u}^{1}+\left(\mathbf{u}^{1} \cdot \nabla\right) \mathbf{u}^{0}+R\left(\theta^{1} \nabla \ln \rho^{0}+\theta^{0} \nabla \ln ^{\prime} \rho^{0} \rho^{1}+\nabla \theta^{1}\right)+f_{1}^{0}=0, \\
\partial_{t} \theta^{1}+\left(\mathbf{u}^{0} \cdot \nabla\right) \theta^{1}+\left(\mathbf{u}^{1} \cdot \nabla\right) \theta^{0}+R \theta^{0} \operatorname{div} \mathbf{u}^{1}+R \theta^{1} \operatorname{div} \mathbf{u}^{0}+f_{2}^{0} \operatorname{div} \mathbf{u}^{0}+f_{3}^{0}\left(\mathbf{u}^{0} \cdot \nabla\right) \theta^{0}=0, \\
\left(\rho^{1}, \mathbf{u}^{1}, \theta^{1}\right)(t=0)=\left(\rho_{1}, \mathbf{u}_{1}, \theta_{1}\right),
\end{gathered}
$$

where

$$
\left(f_{1}^{0}, f_{2}^{0}\right)=\left.\left(f_{1}, f_{2}\right)\right|_{(\rho, \theta)=\left(\rho^{0}, \theta^{0}\right)} .
$$

Inductively, suppose that $\left(p^{k}, \mathbf{u}^{k}, \theta^{k}\right)_{k \leq j-1}$ are solved already for some $j \geq 2$, from (3.4), we know that $\left(p^{j}, \mathbf{u}^{j}, \theta^{j}\right)$ satisfy the following linear problem:

$$
\begin{gathered}
\partial_{t} \rho^{j}+\sum_{k=0}^{j} \operatorname{div}\left(\rho^{k} \mathbf{u}^{j-k}\right)=0, \\
\partial_{t} \mathbf{u}^{j}+\sum_{k=0}^{j}\left(\mathbf{u}^{k} \cdot \nabla\right) \mathbf{u}^{j-k}+R\left(\theta^{j} \nabla \ln \rho^{0}+\theta^{0} \nabla\left(\ln ^{\prime} \rho^{0} \rho^{j}\right)+\nabla \theta^{j}\right)=-g_{1}^{j-1}, \\
\partial_{t} \theta^{j}+R \sum_{k=0}^{j} \theta^{k} \operatorname{div} \mathbf{u}^{j-k}+\sum_{k=0}^{j}\left(\mathbf{u}^{k} \cdot \nabla\right) \theta^{j-k}=-g_{2}^{j-1}, \\
\left(\rho^{j}, \mathbf{u}^{j}, \theta^{j}\right)(t=0)=\left(\rho_{j}, \mathbf{u}_{j}, \theta_{j}\right) .
\end{gathered}
$$

Thus, in order to determine the profiles $\left(\rho^{\epsilon}, \mathbf{u}^{\epsilon}, \theta^{\epsilon}\right)$, we require to solve the nonlinear problem (3.3) for $\left(\rho^{0}, \mathbf{u}^{0}, \theta^{0}\right)$ and the linear system (3.8).

\subsubsection{Existence and Uniqueness of Solution $\left(\rho^{0}, \mathbf{u}^{0}, \theta^{0}\right)$}

Obviously, (3.3) are nonisentropic and compressible Euler equations. Thus, we recall the following the classical result on the existence of sufficiently regular solutions of the compressible Euler equations, see [15]. 
Proposition 3.1. Assume that $\left(\rho_{0}, \mathbf{u}_{0}, \theta_{0}\right) \in H^{s+1} \cap L^{\infty}\left(\mathbb{R}^{d}\right)$ with $\rho_{0}, \theta_{0} \geq C_{1}>0$ and $s>d / 2+1$. Then, there is a finite time $T \in(0, \infty)$, depending on the $H^{s}$ and $L^{\infty}$ norms of the initial data, such that the Cauchy problem (3.3) has a unique bounded smooth solution $(\rho, \mathbf{u}, \theta) \in C\left([0, T] ; H^{s+1}\right) \cap$ $C^{1}\left([0, T] ; H^{s}\right)$.

\subsubsection{Existence and Uniqueness of Solution $\left(\rho^{j}, \mathbf{u}^{j}, \theta^{j}\right)$ for $j \geq 1$}

Now, let us briefly describe the solvability of $\left(\rho^{j}, \mathbf{u}^{j}, \theta^{j}\right)$ for any $j \geq 1$ from the problem (3.3) and (3.8) provided that we have known $\left(\rho^{k}, \mathbf{u}^{k}, \theta^{k}\right)_{k \leq j-1}$ already. Thus, $\left(\rho^{j}, \mathbf{u}^{j}, \theta^{j}\right)$ satisfy the following linear system:

$$
\begin{gathered}
\partial_{t} \rho^{j}+\operatorname{div}\left(\rho^{0} \mathbf{u}^{j}+\rho^{j} \mathbf{u}^{0}\right)=-\sum_{k=0}^{j-1} \operatorname{div}\left(\rho^{k} \mathbf{u}^{j-k}\right), \\
\partial_{t} \mathbf{u}^{j}+\left(\mathbf{u}^{0} \cdot \nabla\right) \mathbf{u}^{j}+\left(\mathbf{u}^{j} \cdot \nabla\right) \mathbf{u}^{0}+R\left(\theta^{j} \nabla \ln \rho^{0}+\theta^{0} \nabla\left(\ln ^{\prime} \rho^{0} \rho^{j}\right)+\nabla \theta^{j}\right)=G_{1}^{j-1}, \\
\partial_{t} \theta^{j}+R\left(\theta^{0} \operatorname{div} \mathbf{u}^{j}+\theta^{j} \operatorname{div} \mathbf{u}^{0}\right)+\left(\mathbf{u}^{0} \cdot \nabla\right) \theta^{j}+\left(\mathbf{u}^{j} \cdot \nabla\right) \theta^{0}=G_{2}^{j-1}, \\
\left(\rho^{j}, \mathbf{u}^{j}, \theta^{j}\right)(t=0)=\left(\rho_{j}, \mathbf{u}_{j}, \theta_{j}\right)
\end{gathered}
$$

where

$$
\begin{gathered}
G_{1}^{j-1}=-g_{1}^{j-1}-\sum_{k=0}^{j-1}\left(\mathbf{u}^{k} \cdot \nabla\right) \mathbf{u}^{j-k} \\
G_{2}^{j-1}=-g_{2}^{j-1}-R \sum_{k=0}^{j-1} \theta^{k} \operatorname{div} \mathbf{u}^{j-k}-\sum_{k=0}^{j-1}\left(\mathbf{u}^{k} \cdot \nabla\right) \theta^{j-k}
\end{gathered}
$$

It is not difficult to see that the system (3.9) can be rewritten as a symmetrizable hyperbolic system. Thus, by the standard existence theory of local smooth solutions of symmetrizable hyperbolic equations (see [15]), we have

Proposition 3.2. Let $T_{0} \in(0, T]$, and assume that $\left(\rho_{j}, \mathbf{u}_{j}, \theta_{j}\right) \in H^{s} \cap L^{\infty}, s>d / 2+1$. Then, there exists a time interval $\left[0, T_{0}\right]$, such that (3.9) or (3.8) has a unique smooth solution $\left(\rho^{j}, \mathbf{u}^{j}, \theta^{j}\right) \in$ $\cap_{i=0}^{1} C^{i}\left(\left[0, T_{0}\right], H^{s-i}\left(\mathbb{R}^{d}\right)\right)$.

Remark 3.3. In particular, if the initial data is $C^{\infty}$, the solution of (3.9) or (3.8) belongs to $C^{\infty}\left(\left[0, T_{0}\right] \times \mathbb{R}^{d}\right)$.

\section{Convergence to Compressible Euler Equations}

In this section, we are devoted to prove the convergence of system (2.3) to compressible Euler equations. 


\subsection{Derivation of Error Equations}

For any fixed integers $m \geq 1$ and $s_{0}>d / 2+2$, set

$$
\begin{aligned}
& \rho_{a, m}^{\epsilon}(x, t)=\sum_{j=0}^{m} \epsilon^{j} \rho^{j}(x, t), \\
& \mathbf{u}_{a, m}^{\epsilon}(x, t)=\sum_{j=0}^{m} \epsilon^{j} \mathbf{u}^{j}(x, t), \\
& \theta_{a, m}^{e}(x, t)=\sum_{j=0}^{m} \epsilon^{j} \theta^{j}(x, t),
\end{aligned}
$$

with $\left(\rho^{j}, \mathbf{u}^{j}, \theta^{j}\right)$ being given by Proposition 3.2. From the asymptotic analysis of Section 3.1, we know that $\left(\rho_{a, m}^{\epsilon}, \mathbf{u}_{a, m}^{e}, \theta_{a, m}^{e}\right)$ satisfy the following problem:

$$
\begin{gathered}
\partial_{t} \rho_{a, m}^{\epsilon}+\operatorname{div}\left(\rho_{a, m}^{\epsilon} \mathbf{u}_{a, m}^{\epsilon}\right)=R_{\rho \prime}^{\epsilon} \\
\partial_{t} \mathbf{u}_{a, m}^{\epsilon}+\left(\mathbf{u}_{a, m}^{\epsilon} \cdot \nabla\right) \mathbf{u}_{a, m}^{\epsilon}+\frac{R \theta_{a, m}^{\epsilon}}{\rho_{a, m}^{\epsilon}} \nabla \rho_{a, m}^{\epsilon}+\left(R+f_{1 a, m} \epsilon\right) \nabla \theta_{a, m}^{\epsilon}=R_{\mathbf{u}^{\prime}}^{\epsilon} \\
\partial_{t} \theta_{a, m}^{\epsilon}+\left(R \theta_{a, m}^{\epsilon}+f_{2 a, m} \epsilon\right) \operatorname{div} \mathbf{u}_{a, m}^{\epsilon}+\left(\mathbf{u}_{a, m}^{\epsilon} \cdot \nabla\right) \theta_{a, m}^{\epsilon}=R_{\theta^{\prime}}^{\epsilon} \\
\left.\left(\rho_{a, m}^{\epsilon}, \mathbf{u}_{a, m}^{\epsilon}, \theta_{a, m}^{\epsilon}\right)\right|_{t=0}=\sum_{j=0}^{m} \epsilon^{j}\left(\rho^{j}, \mathbf{u}^{j}, \theta^{j}\right)(x, 0),
\end{gathered}
$$

where

$$
\left(f_{1 a, m}, f_{2 a, m}\right)=\left(f_{1}, f_{2}\right)\left(\rho_{a, m}^{\epsilon}, \theta_{a, m}^{e}\right)
$$

and the remainders $R_{\rho}^{\epsilon}, R_{\mathbf{u}}^{\epsilon}$, and $R_{\theta}^{\epsilon}$ satisfy

$$
\sup _{0 \leq t \leq T_{0}}\left\|\left(R_{\rho}^{\epsilon}, R_{\mathbf{u}^{\prime}}^{\epsilon}, R_{\theta}^{\epsilon}\right)\right\|_{H^{s_{0}}}<M \epsilon^{m}
$$

for some constant $M>0$ independent of $\epsilon$.

Now, we let $\left(\rho^{\epsilon}, \mathbf{u}^{\epsilon}, \theta^{\epsilon}\right)$ be the smooth solution to the system (2.3) and denote

$$
\left(N^{\epsilon}, U^{\epsilon}, \Theta^{\epsilon}\right)=\left(\rho^{\epsilon}-\rho_{a, m}^{\epsilon}, \mathbf{u}^{\epsilon}-\mathbf{u}_{a, m}^{\epsilon}, \theta^{\epsilon}-\theta_{a, m}^{\epsilon}\right) .
$$


Obviously, $\left(N^{\epsilon}, U^{\epsilon}, \Theta^{\epsilon}\right)$ satisfy the following problem:

$$
\begin{aligned}
& \partial_{t} N^{\epsilon}+\operatorname{div}\left(N^{\epsilon}\left(U^{\epsilon}+\mathbf{u}_{a, m}^{\epsilon}\right)+\rho_{a, m}^{\epsilon} U^{\epsilon}\right)=-R_{\rho \prime}^{\epsilon} \\
& \partial_{t} U^{\epsilon}+\left(\left(U^{\epsilon}+\mathbf{u}_{a, m}^{\epsilon}\right) \cdot \nabla\right) U^{\epsilon}+\frac{R\left(\Theta^{\epsilon}+\theta_{a, m}^{\epsilon}\right)}{N^{\epsilon}+\rho_{a, m}^{\epsilon}} \nabla N^{\epsilon}+\left(R+f_{1}^{\epsilon}\right) \nabla \Theta^{\epsilon}+\left(U^{\epsilon} \cdot \nabla\right) \mathbf{u}_{a, m}^{\epsilon} \\
&+\frac{R \rho_{a, m}^{\epsilon} \Theta^{\epsilon}-R N^{\epsilon} \theta_{a, m}^{\epsilon}}{\rho_{a, m}^{\epsilon}\left(N^{\epsilon}+\rho_{a, m}^{\epsilon}\right)} \nabla \rho_{a, m}^{\epsilon}+\left(f_{1}^{\epsilon}-f_{1 a, m}\right) \nabla \theta_{a, m}^{\epsilon} \epsilon=-R_{\mathbf{u}^{\prime}}^{\epsilon} \\
& \partial_{t} \Theta^{\epsilon}+\left(R\left(\Theta^{\epsilon}+\theta_{a, m}^{\epsilon}\right)+f_{2}^{\epsilon} \epsilon\right) \operatorname{div} U^{\epsilon}+\left(U^{\epsilon}+\mathbf{u}_{a, m}^{\epsilon}\right) \cdot \nabla \Theta^{\epsilon} \\
&+\left(R \Theta^{\epsilon}+\left(f_{2}^{\epsilon}-f_{2 a, m} \epsilon\right)\right) \operatorname{div} \mathbf{u}_{a, m}^{\epsilon}+\left(U^{\epsilon} \cdot \nabla\right) \theta_{a, m}^{\epsilon}=-R_{\theta^{\prime}}^{\epsilon} \\
&\left.\left(N^{\epsilon}, U^{\epsilon}, \Theta^{\epsilon}\right)\right|_{t=0}=\left(N_{0}^{\epsilon}, U_{0}^{\epsilon}, \Theta_{0}^{\epsilon}\right),
\end{aligned}
$$

where

$$
\begin{gathered}
\left(f_{1}^{\epsilon}, f_{2}^{\epsilon}\right)=\left(f_{1}, f_{2}\right)\left(N^{\epsilon}+\rho_{a, m}^{\epsilon}, \Theta^{\epsilon}+\theta_{a, m}^{\epsilon}\right), \\
\left(N_{0}^{\epsilon}, U_{0}^{\epsilon}, \Theta_{0}^{\epsilon}\right)=\left(\rho_{0}^{\epsilon}-\rho_{a, m}^{\epsilon}(x, 0), \mathbf{u}_{0}^{\epsilon}-\mathbf{u}_{a, m}^{\epsilon}(x, 0), \theta_{0}^{e}-\theta_{a, m}^{\epsilon}(x, 0)\right) .
\end{gathered}
$$

Set

$$
\begin{aligned}
& V^{\epsilon}=\left(N^{\epsilon}, U^{\epsilon}, \Theta^{\epsilon}\right)^{T}, \\
& \tilde{A}_{j}\left(V^{\epsilon}\right)=\left(U^{\epsilon}+\mathbf{u}_{a, m}^{\epsilon}\right) I_{(d+2) \times(d+2)}+\left(\begin{array}{ccc}
0 & \left(N^{\epsilon}+\rho_{a, m}^{e}\right) e_{j}^{T} & 0 \\
\frac{R\left(\Theta^{\epsilon}+\theta_{a, m}^{\epsilon}\right)}{N^{\epsilon}+\rho_{a, m}^{\epsilon}} e_{j} & 0 & \left(R+f_{1}^{\epsilon} \epsilon\right) e_{j} \\
0 & {\left[R\left(\Theta^{\epsilon}+\theta_{a, m}^{\epsilon}\right)+f_{2}^{\epsilon} \epsilon\right] e_{j}^{T}} & 0
\end{array}\right), \\
& \mathscr{H}_{1}\left(V^{\epsilon}\right)=\left(\begin{array}{c}
N^{\epsilon} \operatorname{div} \mathbf{u}_{a, m}^{\epsilon}+U^{\epsilon} \cdot \nabla \rho_{a, m}^{\epsilon} \\
\left(U^{\epsilon} \cdot \nabla\right) \mathbf{u}_{a, m}^{\epsilon}+\frac{R \rho_{a, m}^{\epsilon} \Theta^{\epsilon}-R N^{\epsilon} \theta_{a, m}^{\epsilon}}{\rho\left(N^{\epsilon}+\rho_{a, m}^{\epsilon}\right)} \nabla \rho_{a, m}^{\epsilon} \\
R \Theta^{\epsilon} \operatorname{div} \mathbf{u}_{a, m}^{\epsilon}+U^{\epsilon} \cdot \nabla \theta_{a, m}^{\epsilon}
\end{array}\right) \\
& \mathscr{H}_{2}\left(V^{\epsilon}\right)=\left(\begin{array}{c}
0 \\
\left(f_{1}^{\epsilon}-f_{1 a, m}\right) \nabla \theta_{a, m}^{\epsilon} \\
\left(f_{2}^{\epsilon}-f_{2 a, m} \epsilon\right) \operatorname{div} \mathbf{u}_{a, m}^{\epsilon}
\end{array}\right), \quad R^{c}=-\left(\begin{array}{c}
R_{\rho}^{\epsilon} \\
R_{\mathbf{u}}^{\epsilon} \\
R_{\theta}^{\epsilon}
\end{array}\right), \\
& \left.V^{\epsilon}\right|_{t=0}=\left(N_{0}^{\epsilon}, U_{0}^{\epsilon}, \Theta_{0}^{\epsilon}\right) .
\end{aligned}
$$


Thus, the problem (4.6) for the unknown $V^{\epsilon}$ can be rewritten as

$$
\begin{gathered}
\partial_{t} V^{\epsilon}+\sum_{j=1}^{3} A_{j}\left(V^{\epsilon}\right) \partial_{x_{j}} V^{\epsilon}+\mathscr{\ell}_{1}\left(V^{\epsilon}\right)=-\mathscr{H}_{2}\left(V^{\epsilon}\right) \epsilon+R^{\epsilon}, \\
\left.V^{\epsilon}\right|_{t=0}=V_{0}^{\epsilon}=\left(N_{0}^{\epsilon}, U_{0}^{\epsilon}, \Theta_{0}^{\epsilon}\right) .
\end{gathered}
$$

It is easy to see that the equations of $V^{\epsilon}$ in (4.6) are symmetrizable and hyperbolic if we introduce

$$
A_{0}\left(V^{\epsilon}\right)=\left(\begin{array}{ccc}
\frac{R}{\left(N^{\epsilon}+\rho_{a, m}^{e}\right)^{2}} & 0 & 0 \\
0 & \frac{1}{\Theta^{c}+\theta_{a, m}^{\epsilon}} I_{d \times d} & 0 \\
0 & 0 & \frac{R+f_{1}^{\epsilon} \epsilon}{\left(\Theta^{\epsilon}+\theta_{a, m}^{\epsilon}\right)\left(R\left(\Theta^{\epsilon}+\theta_{a, m}^{e}\right)+f_{2}^{\epsilon} \epsilon\right)}
\end{array}\right),
$$

which is positively definite. When $N^{\epsilon}+\rho_{a, m}^{\epsilon}, \Theta^{\epsilon}+\theta_{a, m}^{\epsilon} \geq C>0$ for $\epsilon \ll 1$, then $\tilde{A}_{j}\left(V^{\epsilon}\right)=$ $A_{0}\left(V^{e}\right) A_{j}\left(V^{c}\right)$ are symmetric for all $1 \leq j \leq d$.

\subsection{Proof of Convergence}

Obviously, the existence and uniqueness of smooth solutions of (2.3) are equivalent to that of (4.6) or (4.9). Then, in order to rigorously justify the convergence of (2.3) to (1.3), it suffices to obtain their uniform estimates with respect to the light speed $c$. This will be done by using iteration techniques for the symmetrizable hyperbolic problem.

More amply, we solve the nonlinear problem (4.9) by the following iteration for linear problems (cf. [15]):

$$
\begin{gathered}
\partial_{t} V^{\epsilon, k+1}+\sum_{j=1}^{d} A_{j}\left(V^{\epsilon, k}\right) \partial_{x_{j}} V^{\epsilon, k+1}+\mathscr{L}_{1}\left(V^{\epsilon, k}\right)=-\mathscr{L}_{2}\left(V^{\epsilon, k}\right) c^{-3}+R^{\epsilon}, \\
\left.V^{\epsilon, k+1}\right|_{t=0}=V_{0}^{\epsilon},
\end{gathered}
$$

with

$$
V^{\epsilon, 0}(x, t)=V_{0}^{\epsilon}(x)
$$

To study the problem (4.9) and (4.11), we introduce the Sobolev's norms:

$$
\|V(t)\|_{l}=\left(\sum_{|\alpha| \leq l}\left\|\partial_{x}^{\alpha} V(t)\right\|_{L^{2}\left(\mathbb{R}^{d}\right)}^{2}\right)^{1 / 2}, \quad\|V(t)\|_{l, T}=\sup _{0 \leq t \leq T}\|V(t)\|_{l}, \quad l \in \mathbb{N}^{*} .
$$

The key point for proving the convergence as $\epsilon \rightarrow 0$ is the following a priori estimate. 
Lemma 4.1. Let $s$ and $l$ be two integers such that $d / 2+1<l \leq s$. Assume that

$$
\left\|V_{0}^{\epsilon}\right\|_{l} \leq M_{1} \epsilon^{m}
$$

for some constant $M_{1}>0$ independent of $\epsilon$. Then, there exist constants $M_{2}>0, M_{3}>0, \epsilon_{0}>0$, and $T_{1} \in\left(0, T_{0}\right]$, such that for all $\epsilon \leq \epsilon_{0}$ the solutions $V^{\epsilon, k}$ of (4.11) satisfy

$$
\begin{gathered}
\left\|V^{\epsilon, k}\right\|_{l, T_{1}} \leq M_{2} \epsilon^{m}, \quad \forall k \in \mathbb{N}, \\
\left\|\partial_{t} V^{\epsilon, k}\right\|_{l-1, T_{1}} \leq M_{3} \epsilon^{m}, \quad \forall k \in \mathbb{N} .
\end{gathered}
$$

Proof. Let $\alpha \in \mathbb{N}^{d}$ with $|\alpha| \leq l$. We define $V_{\alpha}^{\epsilon, k}$ by $V_{\alpha}^{\epsilon, k}=\partial_{x}^{\alpha} V^{\epsilon, k}$; thus, it is not difficult to know that $\alpha \in \mathbb{N}^{d}$ satisfies the following problem:

$$
\begin{gathered}
A_{0}\left(V^{\epsilon, k}\right) \partial_{t} V_{\alpha}^{\epsilon, k+1}+\sum_{j=1}^{d} A_{0}\left(V^{\epsilon, k}\right) \tilde{A}_{j}\left(V^{\epsilon, k}\right) \partial_{x_{j}} V_{\alpha}^{\epsilon, k+1}=\mathcal{R}_{\alpha}^{\epsilon, k}, \\
\left.V_{\alpha}^{\epsilon, k+1}\right|_{t=0}=\partial_{x}^{\alpha} V_{0}^{\epsilon}
\end{gathered}
$$

where $\mathcal{R}_{\alpha}^{\epsilon, k}$ is defined by

$$
\begin{aligned}
\mathcal{R}_{\alpha}^{\epsilon, k}= & A_{0}\left(V^{\epsilon, k}\right) \partial_{x}^{\alpha}\left(-\mathscr{L}_{1}\left(V^{\epsilon, k}\right)-\mathscr{H}_{2}\left(V^{\epsilon, k}\right) \epsilon+R^{c}\right) \\
& +\sum_{j=1}^{d} A_{0}\left(V^{\epsilon, k}\right)\left(A_{j}\left(V^{\epsilon, k}\right) V_{\alpha}^{\epsilon, k+1}-\partial_{x}^{\alpha}\left(A_{j}\left(V^{\epsilon, k}\right) V^{\epsilon, k+1}\right)\right) .
\end{aligned}
$$

Estimates (4.15)-(4.16) are obviously true for $k=0$ with any $T_{1}>0$. By induction on $k$, assume (4.15)-(4.16) hold for some $k \geq 1$ where $M_{2}>0$ and $T_{1}>0$ are to $\mathrm{b}$ fixed, and we want to obtain (4.15) for $k+1$, that is:

$$
\left\|V^{\epsilon, k+1}\right\|_{l, T_{1}} \leq M_{2} \epsilon^{m}
$$

which implies, together with $(4.11)_{1}$, that

$$
\left\|\partial_{t} V^{\epsilon, k+1}\right\|_{l-1, T_{1}} \leq M_{3} \epsilon^{m}
$$

In what follows we let $M_{i}(i \geq 4)$ be various positive constants independent of $\epsilon, k \in \mathbb{N}, M_{2}$, and $M_{3}$. 
Equation (4.15) implies that the matrix $A_{0}\left(V^{e, k}\right)$ is positively definite, uniformly with respect to $\epsilon, k \in \mathbb{N}, M_{2}$, and

$$
\left\|V^{\epsilon, k}\right\|_{l, T_{1}} \leq 1, \quad\left\|\partial_{t} V^{\epsilon, k}\right\|_{l-1, T_{1}} \leq 1,
$$

for all $\epsilon \leq \epsilon_{0}$ with some $\epsilon_{0}>0$. Because $l>d / 2+1$, we obtain

$$
\operatorname{div} A_{j}\left(V^{\epsilon, k}\right)=\partial_{t} A_{0}\left(V^{\epsilon, k}\right)+\sum_{j=1}^{d} \partial_{x_{j}} A_{j}\left(V^{\epsilon, k}\right)
$$

satisfying

$$
\left\|\operatorname{div} A_{j}\left(V^{\epsilon, k}\right)\right\|_{L^{\infty}\left(\mathbb{R}^{d} \times\left[0, T_{1}\right]\right)} \leq M_{4} .
$$

Employing the classical energy estimate of symmetric hyperbolic equations to the problem $(4.17)_{1}$, we can obtain

$$
\sup _{0 \leq t \leq T_{1}}\left\|V_{\alpha}^{\epsilon, k+1}\right\|_{L^{2}\left(\mathbb{R}^{d}\right)} \leq M_{5} e^{M_{5} T_{1}}\left(\left\|\partial_{x}^{\alpha} V_{0}^{\epsilon}\right\|_{L^{2}\left(\mathbb{R}^{d}\right)}+\int_{0}^{T_{1}}\left\|\mathcal{R}_{\alpha}^{\epsilon, k}(\tau)\right\|_{L^{2}\left(\mathbb{R}^{d}\right)} d \tau\right)
$$

By the definition of $\mathcal{R}_{\alpha}^{\epsilon, k}$ in (4.18), the classical Moser-type inequality (1.5), (1.7), and Sobolev's embedding lemma with $l>d / 2+1$, we deduce that

$$
\int_{0}^{T_{1}}\left\|\mathcal{R}_{\alpha}^{\epsilon, k}(\tau)\right\|_{L^{2}\left(\mathbb{R}^{d}\right)} d \tau \leq C\left(M_{2}\right) T_{1}\left(\epsilon^{m}+\left\|V^{\epsilon, k+1}\right\|_{l, T_{1}}\right)
$$

Here the constant $C\left(M_{2}\right)>0$ may depend on $M_{2}$. Now, substituting (4.25) into (4.24) and using (4.14), one gets

$$
\left\|V_{\alpha}^{\epsilon, k+1}\right\|_{L^{2}\left(\mathbb{R}^{d}\right)} \leq M_{5} e^{M_{5} T_{1}}\left(M_{1}+C\left(M_{2}\right) T_{1}\right) \epsilon^{m}+M_{5} e^{M_{5} T_{1}} C\left(M_{2}\right) T_{1}\left\|V_{\alpha}^{\epsilon, k+1}\right\|_{L^{2}\left(\mathbb{R}^{d}\right)} .
$$

Now, we choose $T_{1}>0$ such that

$$
e^{M_{5} T_{1}} \leq 2, \quad C\left(M_{2}\right) T_{1} \leq 1, \quad M_{5} e^{M_{5} T_{1}} C\left(M_{2}\right) T_{1} \leq \frac{1}{2}
$$

Then

$$
\left\|V^{c, k+1}\right\|_{l, T_{1}} \leq M_{2} \epsilon^{m}
$$

with $M_{2}=4 M_{5}\left(M_{1}+1\right)$. The proof of Lemma 4.1 is complete.

Returning to the problem (2.3) and (4.6), we conclude the following. 
Theorem 4.2. For any fixed integers $s>d / 2+1$, suppose that

$$
\left\|\left(\rho_{0}^{\epsilon}, \mathbf{u}_{0}^{\epsilon}, \theta_{0}^{\epsilon}\right)-\sum_{j=0}^{m} \epsilon^{j}\left(\rho_{j}, \mathbf{u}_{j}, \theta_{j}\right)\right\|_{s} \leq M \epsilon^{m} .
$$

Then the solution of (2.3) satisfies

$$
\left\|\left(\rho^{\epsilon}, \mathbf{u}^{\epsilon}, \theta^{\epsilon}\right)-\sum_{j=0}^{m} \epsilon^{j}\left(\rho^{j}, \mathbf{u}^{j}, \theta^{j}\right)\right\|_{s, T_{1}} \leq M \epsilon^{m},
$$

where $M>0$ is a constant independent of $\epsilon$.

Proof. First, the uniform estimates (4.15)-(4.16), together with (4.11), yield the bound of the sequence $\left\{V^{\epsilon, k}\right\}_{k \in \mathbb{N}}$ in $L^{\infty}\left(\left[0, T_{1}\right], H^{s}\left(\mathbb{R}^{d}\right)\right) \cap W^{1, \infty}\left(\left[0, T_{1}\right]\right.$, and $\left.H^{s-1}\left(\mathbb{R}^{d}\right)\right)$. Then Aubin's lemma implies that $\left\{V^{\epsilon, k}\right\}_{k \in \mathbb{N}}$ is compact in $C\left(\left[0, T_{1}\right], C^{1}\left(\mathbb{R}^{d}\right)\right)$. Hence, up to a subsequence, $\left\{V^{\epsilon, k}\right\}_{k \in \mathbb{N}}$ convergence to some $V^{\epsilon}$ in the space $C\left(\left[0, T_{1}\right], C^{1}\left(\mathbb{R}^{d}\right)\right)$ as $k \rightarrow \infty$. Combining this with the bound results (4.15)-(4.16), we have $V^{\epsilon} \in C\left(\left[0, T_{1}\right], H^{s}\left(\mathbb{R}^{d}\right)\right) \cap \operatorname{Lip}\left(\left[0, T_{1}\right]\right.$, and $H^{s-1}\left(\mathbb{R}^{d}\right)$ ). Furthermore, a similar argument as in [15] (see Theorem 2.1(b)) gives $V^{e} \in$ $C^{1}\left(\left[0, T_{1}\right], H^{s-1}\left(\mathbb{R}^{d}\right)\right)$. Passing to the limit $k \rightarrow \infty$ in the system (4.11) shows that $V^{\epsilon}$ is a classical solution to the problem (4.6). The uniqueness implies the convergence of the whole sequence $\left\{V^{\epsilon, k}\right\}_{k \in \mathbb{N}}$ to $V^{\epsilon}$.

Finally, the estimate (4.30) can be easily derived from the estimate (4.24). This ends the proof of Theorem 4.2 .

\section{Acknowledgments}

The authors cordially acknowledge partial support from the National Science Foundation of China (Grant no. 10771099,10901011), the Beijing Science Foundation (Grant no. 1082001), Beijing Municipal Commission of Education, Funding Project for Academic Human Resources Development in Institutions of High Learning Under the Jurisdiction of Beijing Municipality (PHR200906103), and Research Initiation Project for High-level Talents (no. 201025) of North China University of Water Resources and Electric Power.

\section{References}

[1] D. Mihalas and B. W. Mihalas, Foundations of Radiation Hydrodynamics, Oxford University Press, New York, NY, USA, 1984.

[2] G. C. Pomraning, The Equations of Radiation Hydrodynamics, Pergamon Press, Oxford, UK, 1973.

[3] J. I. Castor, Radiation Hydrodynamics, Cambridge University Press, Cambridge, Mass, USA, 2004.

[4] A. M. Anile, A. M. Blokhin, and Yu. L. Trakhinin, "Investigation of a mathematical model for radiation hydrodynamics," Zeitschrift für Angewandte Mathematik und Physik, vol. 50, no. 5, pp. 677-697, 1999.

[5] S. Jiang and W. Sun, "A second-order BGK scheme for the equations of radiation hydrodynamics," International Journal for Numerical Methods in Fluids, vol. 53, no. 3, pp. 391-416, 2007.

[6] C. Rohde and W.-A. Yong, "The nonrelativistic limit in radiation hydrodynamics. I. Weak entropy solutions for a model problem," Journal of Differential Equations, vol. 234, no. 1, pp. 91-109, 2007.

[7] L. P. Horwitz and F. C. Rotbart, "Nonrelativistic limit of relativistic quantum mechanics," Physical Review D, vol. 24, no. 8, pp. 2127-2131, 1981. 
[8] N. Masmoudi and K. Nakanishi, "Nonrelativistic limit from Maxwell-Klein-Gordon and MaxwellDirac to Poisson-Schrödinger," International Mathematics Research Notices, vol. 2003, no. 13, pp. 697734, 2003.

[9] S. Calogero and H. Lee, "The non-relativistic limit of the Nordström-Vlasov system," Communications in Mathematical Sciences, vol. 2, no. 1, pp. 19-34, 2004.

[10] Y. Li and Y. Geng, "Non-relativistic global limits of entropy solutions to the isentropic relativistic Euler equations," Zeitschrift für Angewandte Mathematik und Physik, vol. 57, no. 6, pp. 960-983, 2006.

[11] Y.-J. Peng and S. Wang, "Convergence of compressible Euler-Maxwell equations to incompressible Euler equations," Communications in Partial Differential Equations, vol. 33, no. 1-3, pp. 349-376, 2008.

[12] J. Yang and S. Wang, "Convergence of the nonisentropic Euler-Maxwell equations to compressible Euler-Poisson equations," Journal of Mathematical Physics, vol. 50, no. 12, Article ID 123508, 15 pages, 2009.

[13] S. Klainerman and A. Majda, "Singular limits of quasilinear hyperbolic systems with large parameters and the incompressible limit of compressible fluids," Communications on Pure and Applied Mathematics, vol. 34, no. 4, pp. 481-524, 1981.

[14] S. Klainerman and A. Majda, "Compressible and incompressible fluids," Communications on Pure and Applied Mathematics, vol. 35, no. 5, pp. 629-651, 1982.

[15] A. Majda, Compressible Fluid Flow and Systems of Conservation Laws in Several Space Variables, vol. 53 of Applied Mathematical Sciences, Springer, New York, NY, USA, 1984. 